\title{
Le môle nord-sud de Portus. Seconde campagne de fouilles
}

Évelyne Bukowiecki, Milena Mimmo et Charles-Édouard Sauvin

\section{(2) OpenEdition \\ Journals}

Édition électronique

URL : http://journals.openedition.org/cefr/3777

DOI : $10.4000 /$ cefr.3777

ISSN : 2282-5703

Éditeur

École française de Rome

Référence électronique

Évelyne Bukowiecki, Milena Mimmo et Charles-Édouard Sauvin, « Le môle nord-sud de Portus.

Seconde campagne de fouilles ", Chronique des activités archéologiques de l'École française de Rome [En ligne], Italie centrale, mis en ligne le 07 octobre 2019, consulté le 09 octobre 2019. URL : http:// journals.openedition.org/cefr/3777; DOI : 10.4000/cefr.3777

Ce document a été généré automatiquement le 9 octobre 2019.

(c) École française de Rome 


\title{
Le môle nord-sud de Portus. Seconde campagne de fouilles
}

\author{
Évelyne Bukowiecki, Milena Mimmo et Charles-Édouard Sauvin
}

\section{NOTE DE L'AUTEUR}

Ont participé aux travaux de cette seconde campagne qui s'est déroulée du 10 au 28 septembre 2018 : Évelyne Bukowiecki, Bertrand Augier (École française de Rome), Ehsan Behbahani Nia (Université de Strasbourg), Charles Bigo (École Spéciale des Travaux Publics, du bâtiment et de l'industrie), Jorge Cocquyt (Sapienza Università di Roma), Arianna Collari (Sapienza Università di Roma), Charles Davoine (École française de Rome), Rémi Fabro (ArkoD - Humanités Digitales, Lugdunum), Letizia Fanfani (Sapienza Università di Roma), Marta Fedeli (Rome), François Fouriaux (École française de Rome), Ilaria Frumenti (Rome), Francesca Galanti (Sapienza Università di Roma), Federico Gallinucci (Sapienza Università di Roma), Baptiste Gouttenegre (Université Grenoble Alpes), Bianca Magliocchetti (Sapienza Università di Roma), Milena Mimmo, Ascanio Modena Altieri (Sapienza Università di Roma), Emanuele Musa (Università di Roma Tre), Francesco Perotta (Sapienza Università di Roma) et Charles-Édouard Sauvin. Nous tenons à remercier cordialement pour la confiance renouvelée l'ensemble du personnel du Parco Archeologico di Ostia Antica et en particulier sa directrice, Mariarosaria Barbera, l'inspecteur du site de Portus, Luigi Gambaro, Marina Lo Blundo, Stefania Pietrini et Franca Tortora.

Cette chronique résume le rapport d'activité détaillé intitulé « Il molo nord-sud di Portus - Relazione sulla campagna 2019 » et déposé au Parco Archeologico di Ostia Antica et à l'EFR.

1 Les résultats de la première campagne d'étude du môle nord-sud de Portus ${ }^{1}$, qui avaient permis en 2017 d'en identifier les trois phases de construction (fig. 1), ont été vérifiés et approfondis en 2018 par une intervention ciblée (fig. 2) dans la zone de connexion 
entre l'extrémité nord du môle de Claude et sa première extension datée de l'époque sévérienne (secteur C).

Fig. 1 - Restitution schématique des trois phases de construction du môle nord-sud de Portus : phase claudienne, phase sévérienne, phase tardo-antique.

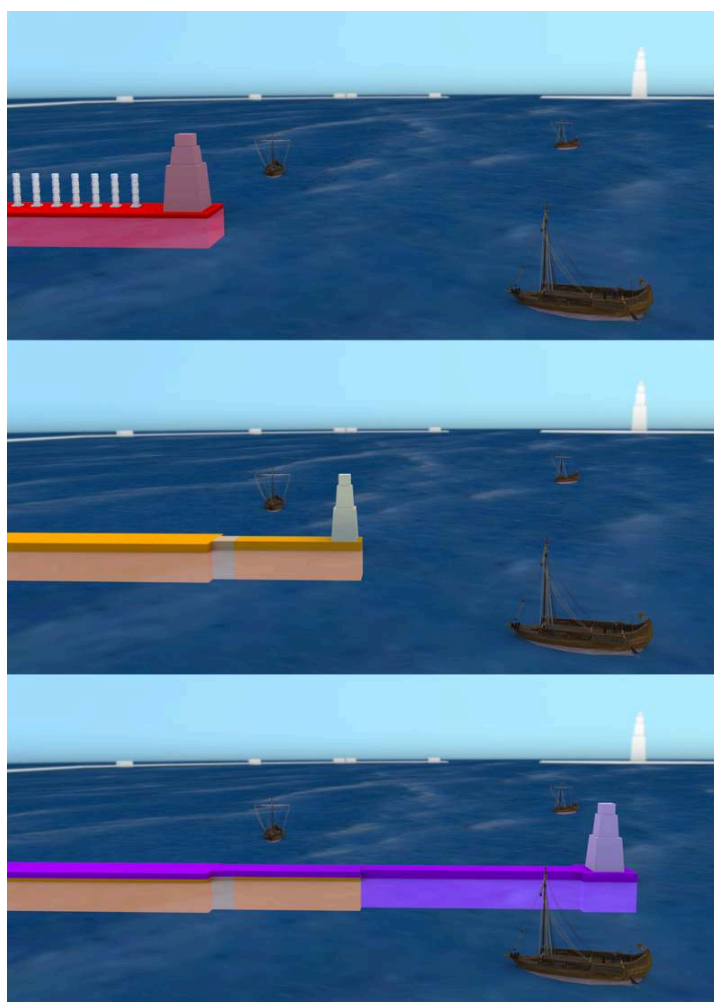

R. Fabro. 
Fig. 2 - Localisation du secteur de fouille (secteur C) ouvert en 2018 sur le môle nord-sud de Portus.

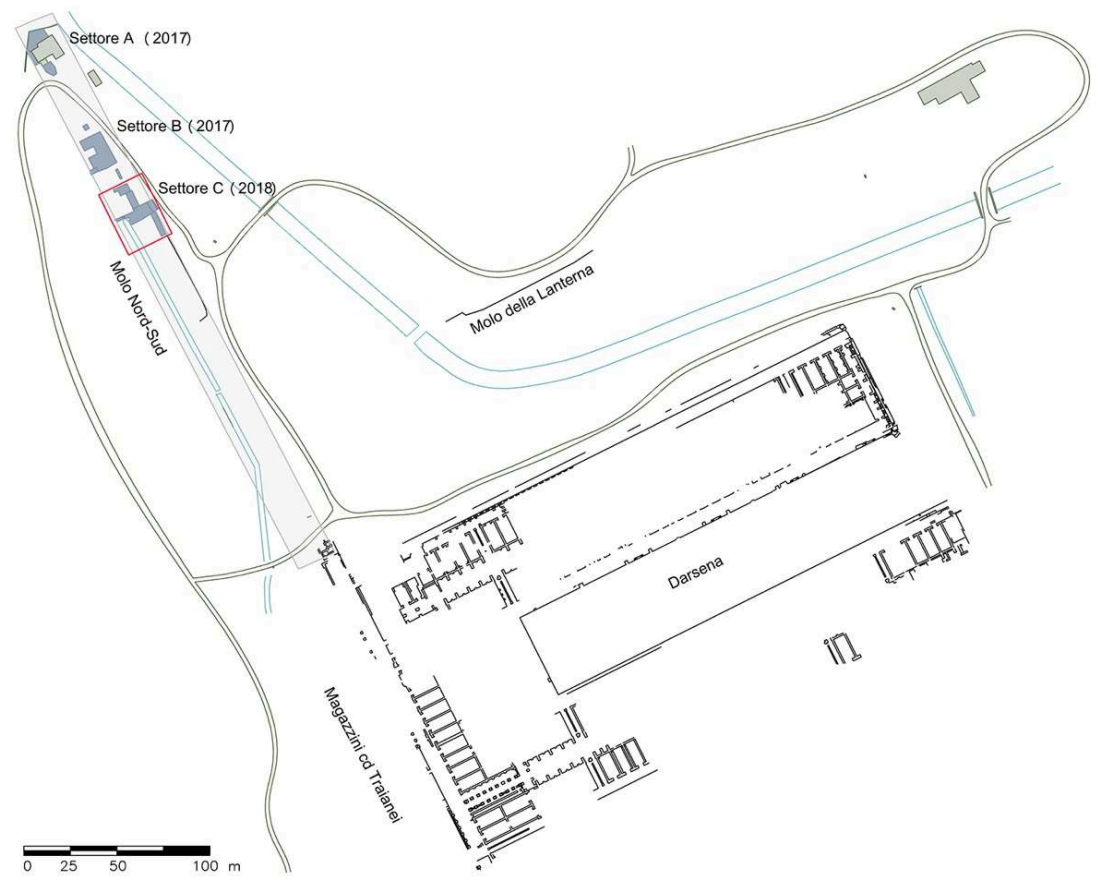

M. Mimmo.

2 L'objectif était de mieux observer les caractéristiques constructives du môle de Claude et de comprendre comment le môle sévérien s'est inséré dans sa continuité. Depuis les observations réalisées en 2017, nous savions déjà qu'il y avait peu d'espoir de retrouver l'appareil décoratif du môle original mais nous pensions au moins atteindre son niveau de fondation afin d'en vérifier la technique de construction. En effet, le môle nord-sud, comme la plupart des chantiers claudiens à Portus ${ }^{2}$, devait être construit en opus caementicium coffré puis surmonté hors terre d'une élévation parementée en opus reticulatum qui permettait de garantir une surélévation des structures au-dessus du niveau de la mer.

Le sondage réalisé en 2018 (fig. 3) au pied de la façade portuaire du môle (Sondage C1) n'a malheureusement pu permettre d'observer ce niveau de fondation désormais recouvert par d'autres structures portuaires datant vraisemblablement de la fin de l'antiquité. 
Fig. 3 - Planimétrie détaillée du secteur C.

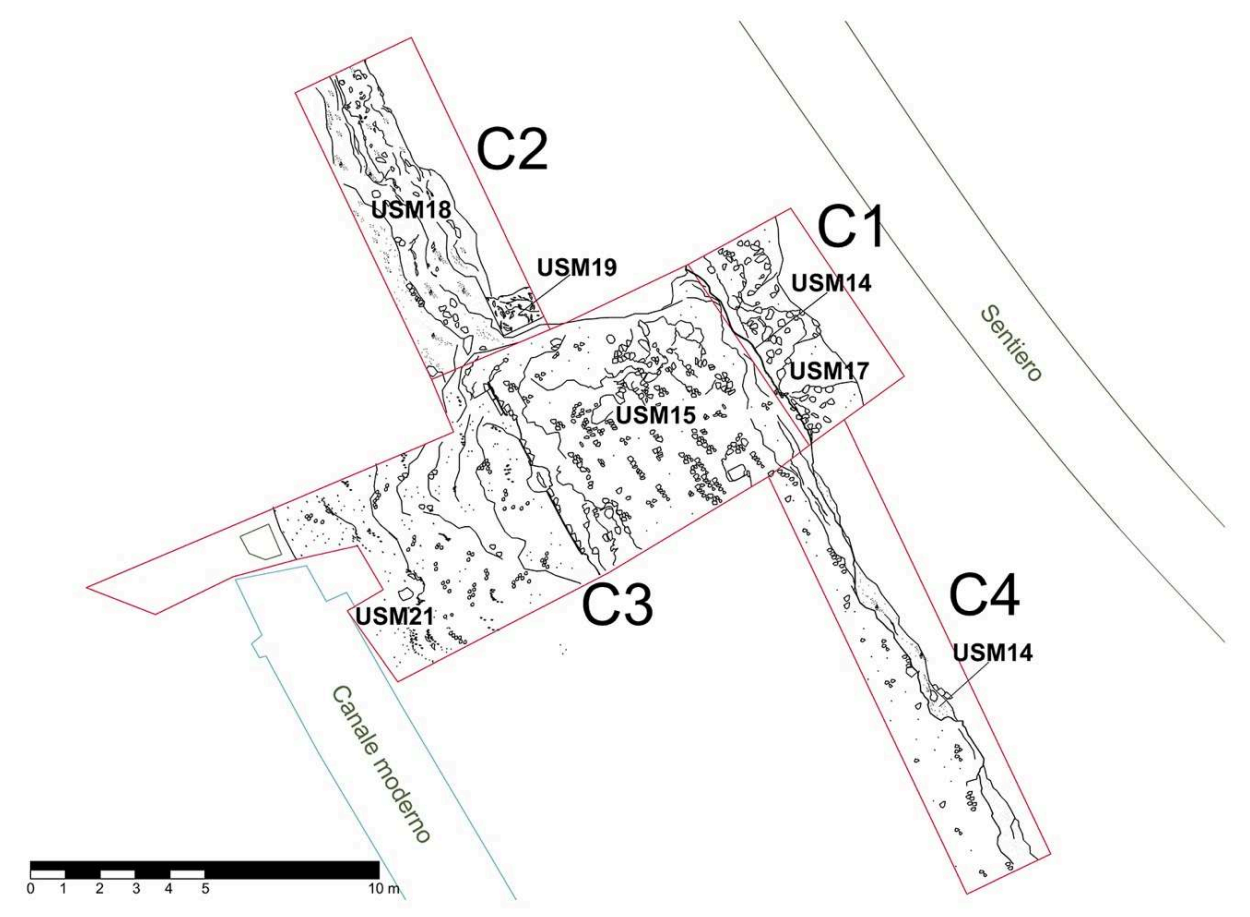

M. Mimmo

4 La zone de contact entre le large môle de Claude (env. $14 \mathrm{~m}$ ) et l'extension sévérienne plus étroite (env. 7-8 m) a été observée également du côté de la façade portuaire (Sondage C2) ; la façade maritime du môle étant devenue inaccessible sur quasiment toute sa longueur à cause de l'implantation au début du siècle dernier d'une canalisation ouverte en ciment. Cette première extension du môle originel appartient à une réorganisation générale de l'époque sévérienne de l'ensemble des quais et des môles de Portus, concomitante aux derniers aménagements du complexe de stockage claudien (les entrepôts dits de Trajan). Ces derniers visaient à potentialiser les capacités de stockage et la création de nouveaux entrepôts au nord-est du site portuaire (les entrepôts dits sévériens). Ainsi, l'élévation aujourd'hui visible du môle de Claude correspond à une reconstruction à l'époque sévérienne (Sondages $\mathrm{C} 3$ et $\mathrm{C} 4$ ), réalisée avec les matériaux et les techniques constructives de l'époque, et intégrant au nord la réalisation de la nouvelle extension du môle (Sondage C2).

\section{Sondage $\mathrm{C} 1$}

5 Situé dans l'angle nord-est du môle de Claude, le sondage $\mathrm{C} 1$ se développe sur $6 \mathrm{~m}$ le long de la façade portuaire et sur une largeur de $3,5 \mathrm{~m}$. L'objectif principal de sa réalisation était, comme déjà mentionné, de retrouver la fondation du môle de Claude dont l'élévation originelle a complètement disparu lors de sa reconstruction à l'époque sévérienne. Même si la fondation claudienne n'a pas été retrouvée, le sondage $\mathrm{C} 1$ a permis toutefois de mettre en évidence une portion d'un aménagement portuaire (USM 17) plus tardif, sans doute contemporain de la dernière phase d'utilisation du port (fig. 4). Cet aménagement, qui n'est pas sans rappeler ceux identifiés par l'équipe espagnole de l'Université de Huelva ${ }^{3}$, contre la façade nord du proche môle de la 
Lanterne, et pour lequel a été proposé un terminus post quem autour $\mathrm{du} \mathrm{V}^{\mathrm{e}}$ siècle apr. J.C., présente deux traces transversales en négatif appartenant à une solide armature en bois permettant de restituer la construction en eau de cette structure. L'USM 17, qui présente un fort dénivelé vers le port ${ }^{4}$ (fig. 5), se situe à une altitude comprise entre $-0,50$ et $-1,90 \mathrm{~m}$, soit environ $1,5 \mathrm{~m}$ plus bas que la partie supérieure du réaménagement sévérien du môle de Claude. Cet aménagement tardif contre la paroi du môle, ainsi que ceux observés contre la paroi du môle de la Lanterne, laissent supposer qu'à cette époque, les grosses embarcations ne pouvaient plus aussi facilement accoster le long des môles pour charger et décharger leurs marchandises et qu'un autre type de navigation, avec peut-être une flotte plus modeste, devait avoir pris le relais.

Fig. 4 - Vue de l'est du sondage $\mathrm{C} 1$ avec la reconstruction sévérienne du môle de Claude (USM 14) et l'aménagement portuaire tardif (USM 17) contre l'USM 14.

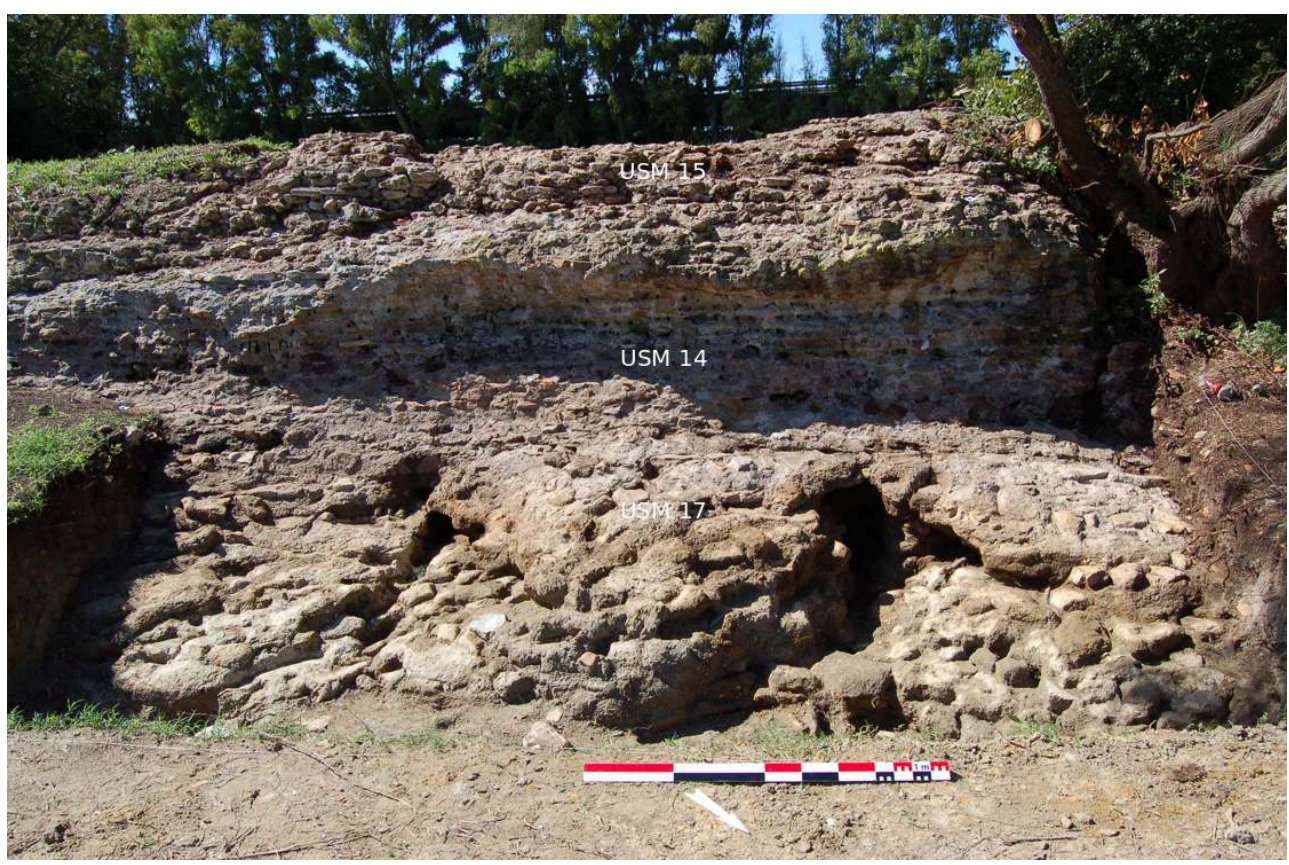

F. Galanti. 
Fig. 5 - Vue du sondage $\mathrm{C} 1$ en fin de fouille : I'US 50 pourrait encore recouvrir une partie de I'USM 17

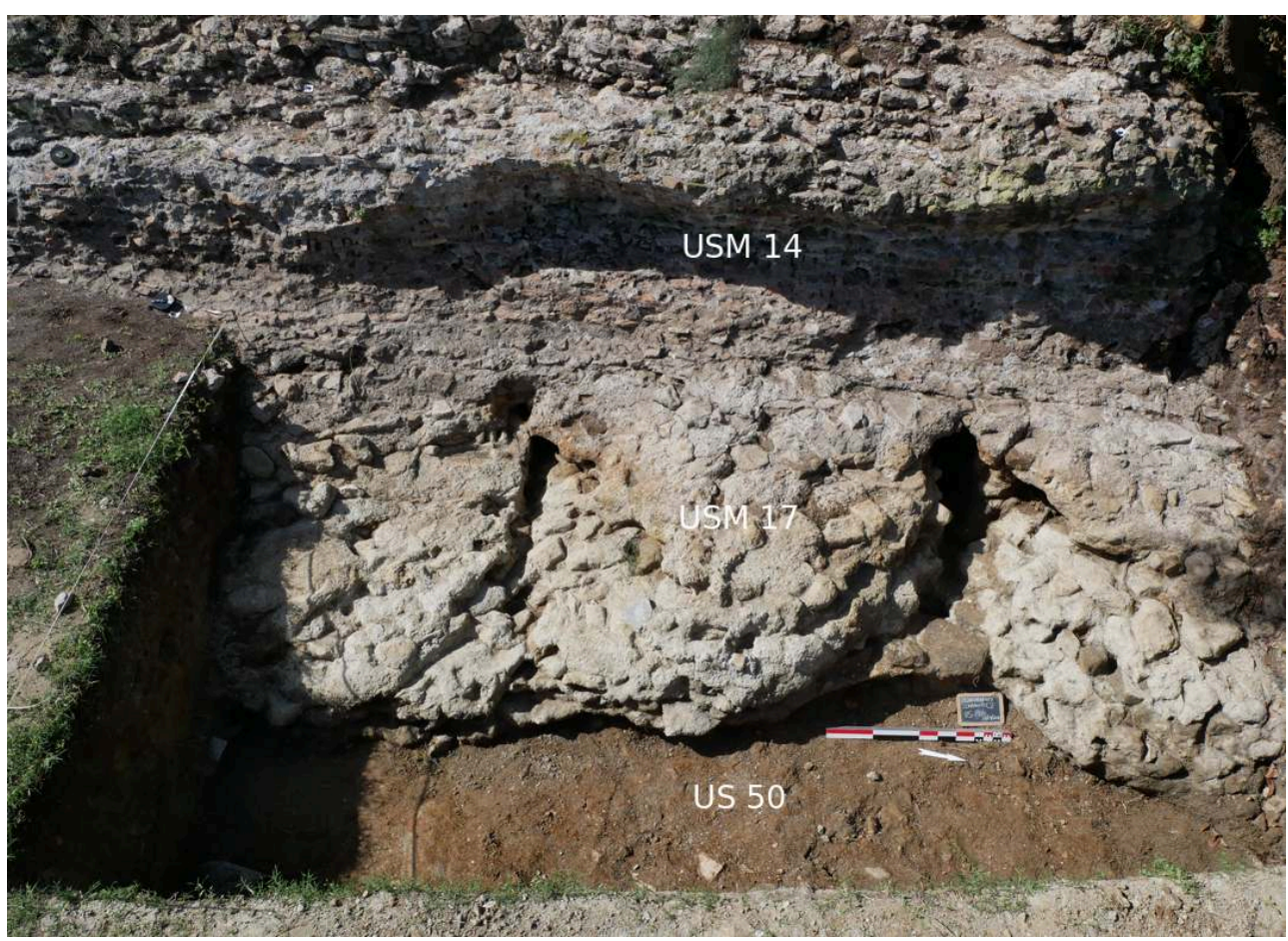

F. Galanti.

6 Sous les premières couches d'époque contemporaine (US 20, US 23, US 25), la stratigraphie $^{5}$ qui recouvrait l'USM 17 (fig. 6) témoigne d'une série de processus de sédimentation en milieu aquatique. La présence massive de malacofaune dans la plupart des unités stratigraphiques identifiées ${ }^{6}$ (US 26, US 24, US 30, US 31, US 47) en est un indice évident. 
Fig. 6 - Coupes stratigraphiques du sondage $\mathrm{C} 1$.
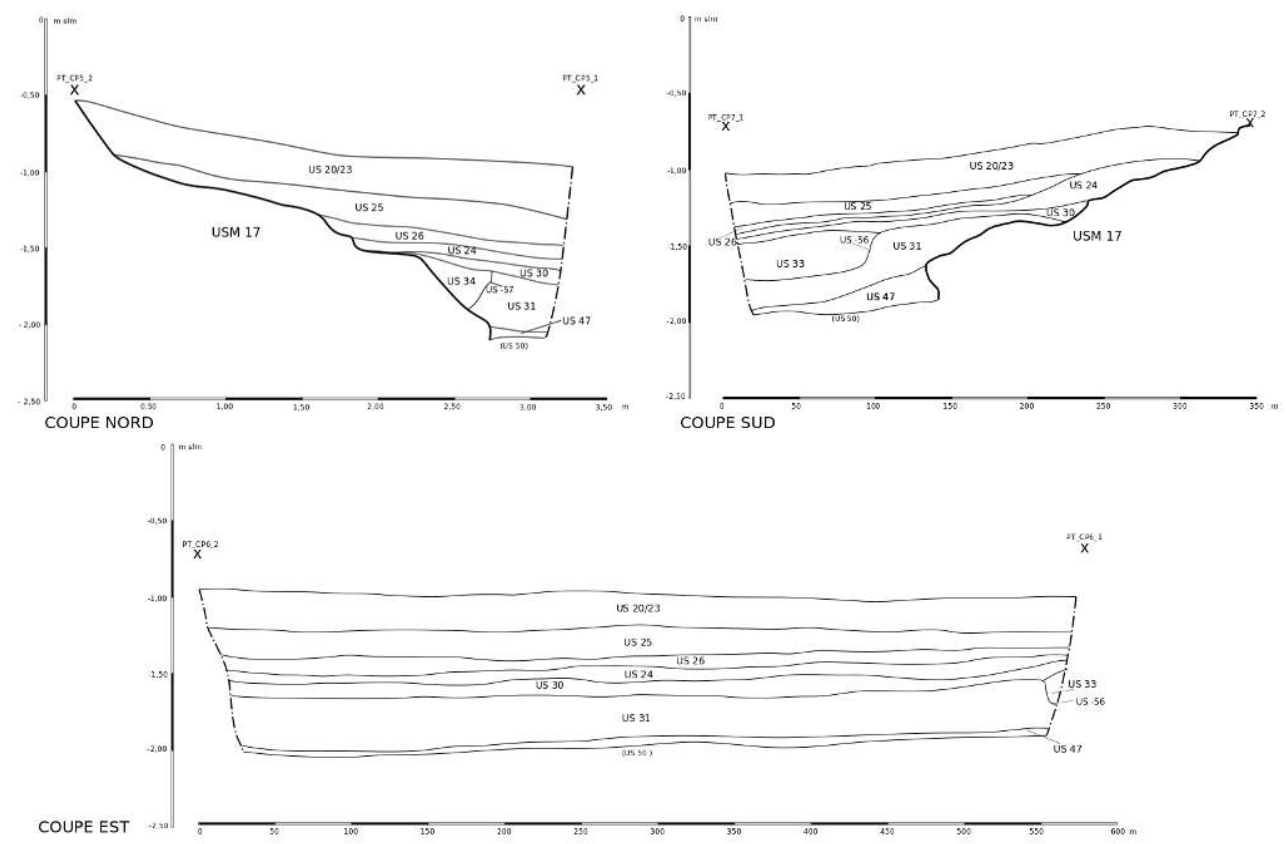

Ch.-É. Sauvin.

7 Comme nous pouvons le constater sur les figure 4 et 5, l'USM 17 s'appuie très solidement sur la maçonnerie de l'USM 14 qui forme la reconstruction sévérienne du môle de Claude. Il est d'ailleurs probable que l'armature en bois ait été insérée volontairement dans la structure précédente comme le suggère la perforation dans la maçonnerie sévérienne constatée sur l'une des deux traces correspondant au négatif des pièces de bois transversales qui constituaient cette armature.

\section{Sondage C2}

8 Le sondage $\mathrm{C} 2$, positionné immédiatement au sud du dernier sondage réalisé en 2017 sur le môle séverien (Sondage B3), a permis de mettre en évidence, toujours du côté de la façade portuaire, la zone d'insertion entre l'élévation sévérienne du môle de Claude, précédemment décrite, et le nouveau môle sévérien (fig. 7). 
Fig. 7 - Vue générale du sondage $C 2$.

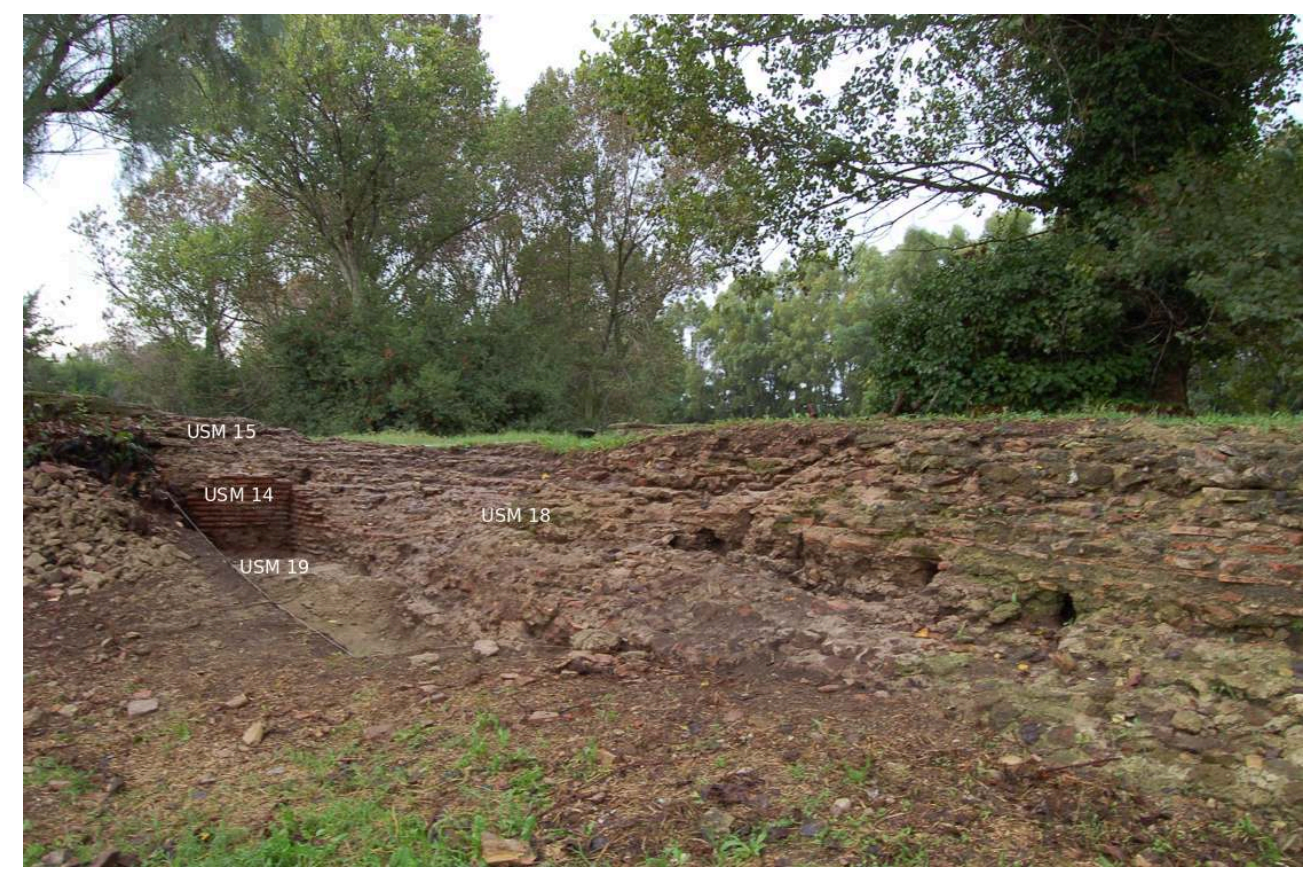

A. Collari.

9 Cette première extension du môle originel se développe vers le nord sur une longueur d'environ 45 mètres et sa largeur médiane se situe entre 7 et 8 mètres. Les traces de l'ossature en bois autour de laquelle le môle sévérien a été construit ${ }^{7}$ sont encore largement visibles sur les structures. Nous avons pu observer et analyser le négatif d'une partie du coffrage extérieur (l'arca), la première rangée des pieux verticaux qui le maintenaient (les destinae) et la rangée supérieure des pieux horizontaux qui consolidaient l'ensemble transversalement (les catenae).

10 Au-delà de la mise au jour de l'angle en maçonnerie de briques entre les deux môles (fig. 8), le sondage $\mathrm{C} 2$ a également permis de mieux comprendre pourquoi les premiers mètres du môle sévérien ne présentent aucune trace d'armature en bois. En effet, nous avons pu observer sous l'élévation de l'extrémité sud du môle, un amas hétérogène de matériaux, liés à un grossier mais puissant mortier de pouzzolane. Ce dernier semble avoir été déposé dans l'eau, sans coffrage, contre la paroi nord du môle précédent, de manière à créer un point d'attache solide pour développer, dans un second temps, l'ossature en bois du môle et y appuyer son élévation. Ce blocage est composé essentiellement de matériaux de réemploi, peut-être provenant de la démolition des structures antérieures, dont certains fragments présentent des traces de chauffe. À la jonction du blocage et de la partie du môle présentant les traces d'une armature en bois, une large cavité de $1,20 \mathrm{~m}$ sur $0,90 \mathrm{~m}$ a été mise au jour (US-58). Il s'agit vraisemblablement du négatif d'une grosse pièce de bois, sans doute une partie d'un tronc d'arbre, qui pouvait empêcher les matériaux déposés en vrac de s'étendre vers le nord et ainsi de les contenir, en attendant la consolidation du mortier de pouzzolane qui les liait. Après décomposition du bois, cette cavité insérée dans la maçonnerie du môle a été remplie de nombreux détritus de nature variable (matériel céramique, matériaux de constructions, malacofaune, lentilles d'argile, etc.). De celle-ci, nous avons également pu observer en détail les négatifs verticaux ${ }^{8}$ laissées par les premières destinae, d'un diamètre constant autour de $15 \mathrm{~cm}$. L'humidité de cette cavité a permis la 
conservation d'un fragment de bois dont l'analyse nous consentira de déterminer le type d'essence utilisée par le chantier sévérien.

Fig. 8 - Sondage C2 : extension sévérienne du môle de Claude.

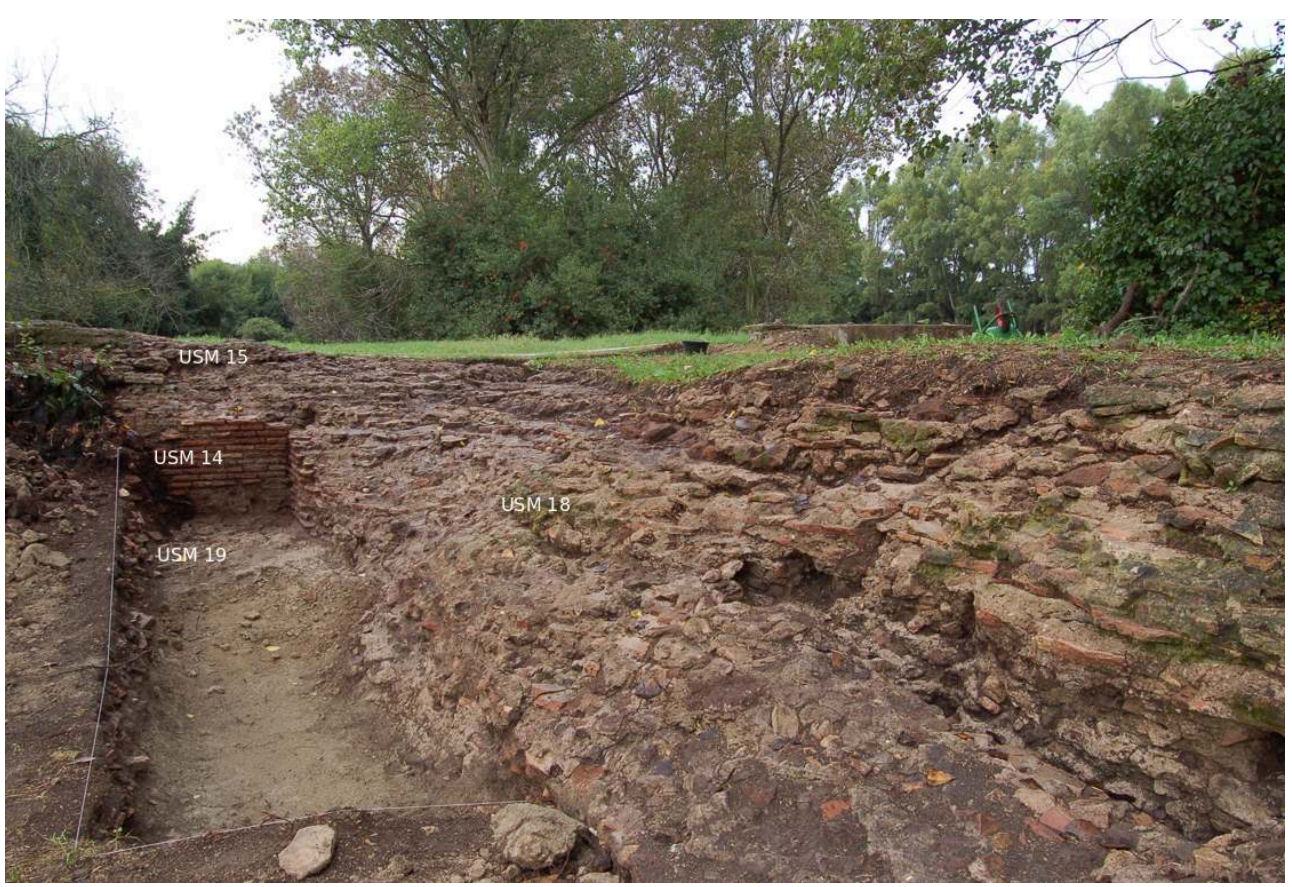

A. Collari.

11 Enfin, une des premières couches contemporaines du sondage C2 (US 27) a révélé du mobilier plutôt insolite, constitué par une réserve importante de munitions de fusil. Ces munitions datent de la seconde guerre mondiale, selon l'expertise des artificiers de Fiumicino qui sont intervenus après la signalisation de notre découverte. Il semblerait qu'à cette époque et dans cet angle des ruines de Portus, un poste d'observation ait été effectivement aménagé.

\section{Sondages C3 et C4}

12 Le sondage C3 (fig. 9) fut limité à un nettoyage approfondi du sommet arasé de l'élévation sévérienne du môle de Claude (USM 14). Cette opération a permis de mettre en évidence un aménagement plus tardif (USM 15) le long de la limite orientale de cette élévation. Dans la partie occidentale, une maçonnerie antérieure à l'USM 15 a été identifiée (USM 21) mais les mauvaises conditions d'observation, limitées par la présence du canal moderne, ne permettent pas de comprendre s'il s'agit de la continuité vers l'ouest de l'élévation sévérienne (USM 14) ou d'une autre maçonnerie précédente, peut-être d'époque claudienne. La poursuite du sondage C3 vers l'ouest, possible sur une très petite largeur dans la zone où le canal moderne est enterré, a cherché à identifier dans cette partie, la façade maritime du môle de Claude, sans succès. En effet, dans cette étroite portion du môle, la construction de la portion souterraine du conduit moderne avait malheureusement endommagé les structures antiques voisines. 
Fig. 9 - Vue vers l'est du sondage C3.

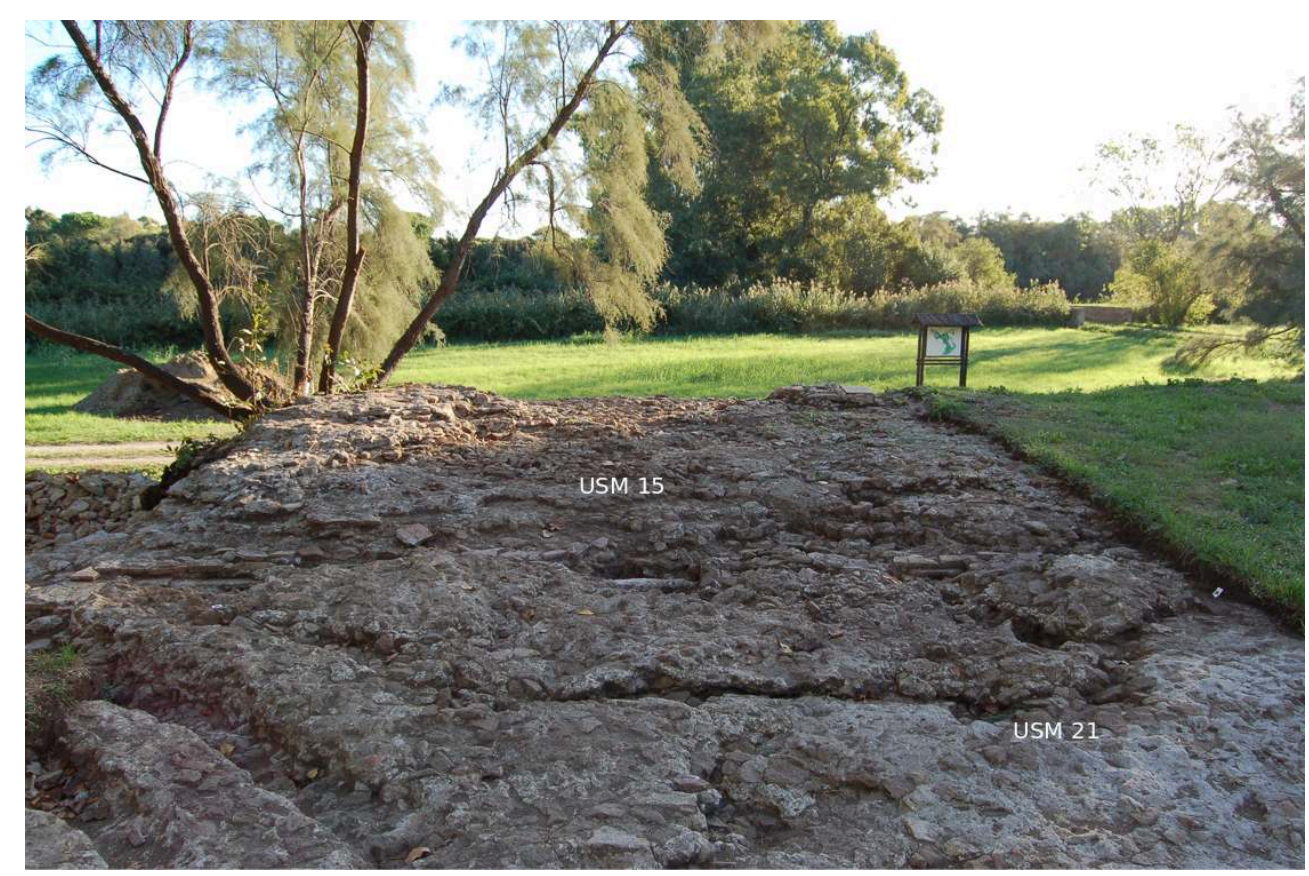

M. Mimmo.

13 Nous continuons à considérer toutefois que l'implantation particulièrement rectiligne de la canalisation en ciment, exactement parallèle au môle de Claude, s'explique par son insertion contre sa façade maritime. Dans ces conditions, celle-ci ne peut plus être observée sur la majeure partie de sa longueur. La dernière chance que nous aurons de pouvoir l'identifier sera d'intervenir autour de la portion initiale du môle de claude ${ }^{9}$, en connexion avec le portique de Claude, là où le canal moderne a déjà opéré un changement de trajectoire vers l'ouest.

Le sondage $\mathrm{C} 4$, situé en continuité des sondages $\mathrm{C} 1$ et $\mathrm{C} 3$, vers le sud, a également été réduit à un nettoyage superficiel livrant l'élévation sévérienne du môle de claude (fig. 10). Nous avons pu alors en analyser les caractéristiques techniques employées, typiques des chantiers de la fin du $\mathrm{II}^{\mathrm{e}}$ et du début du $\mathrm{III}^{\mathrm{e}}$ siècle apr. J.-C., comme le confirme l'utilisation d'une production de fines briques (env. 2,5 cm) en parement, d'assises de réglage en bipédales rouges, également très fins, et d'une proportion massive de fragments de tuf comme caementa. 
Fig. 10 - Vue générale des sondages $\mathrm{C1}, \mathrm{C3}$ et $\mathrm{C} 4$.

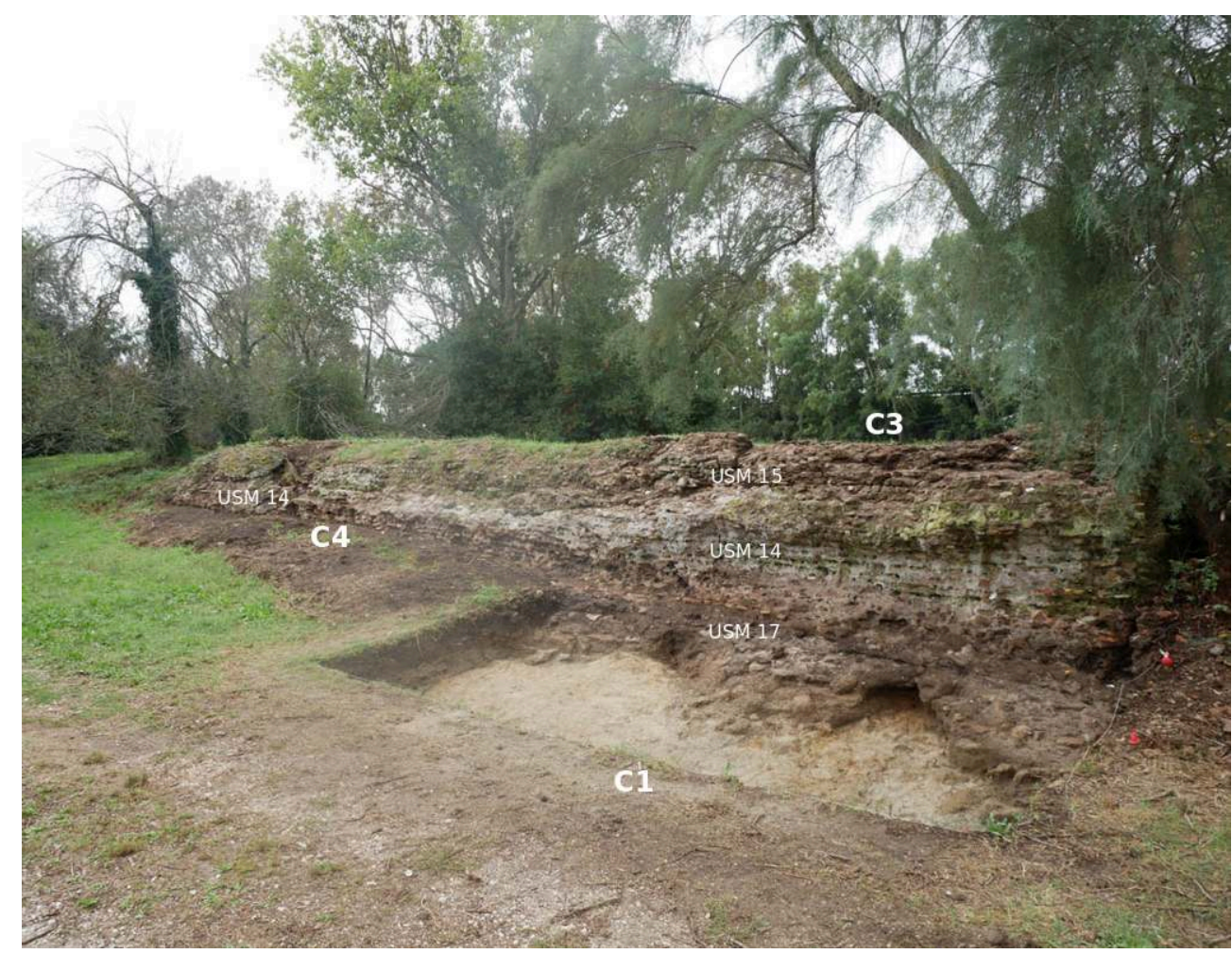

A. Collari.

15 L'absence de cavités liées à une armature interne en bois sur l'ensemble de l'élévation sévérienne, observée entre le sondage $\mathrm{C} 1$ et $\mathrm{C} 4$, témoigne que cette dernière s'appuie directement sur une construction précédente, le môle de Claude. Le mauvais état de conservation des maçonneries, dans cette zone, n'a toutefois pas permis d'intervenir sur le sommet du môle et il n'a donc pas été possible de vérifier si l'aménagement tardif, identifié le long du côté est du môle dans le sondage C3 (USM 15), se poursuivait jusque-là.

\section{Catalogue des timbres sur brique de Portus}

La campagne 2018 a permis également de compléter le recueil initié en 2017 des timbres sur brique retrouvés à Portus. C'est en particulier les timbres provenant de la basilique de Portus et entreposés dans les baraques préfabriquées situées au sud du site, à l'est du cimetière moderne de Fiumicino, qui ont été traités cette année.

Cette opération s'insère dans le projet de catalogue unique des timbres sur briques de Portus en cours de réalisation dans le cadre du protocole d'accord international mentionné à la note 1 ainsi que dans le projet en programmation entre le DAI ${ }^{10}$ de Berlin et le DAI de Rome (BOLARO: Bolli Laterizi di Roma) qui vise à recueillir sous forme de fiche individuelle synthétique liée à une illustration pour chaque timbre (frottis ou photographie) et selon la localisation topographique précise de son lieu de découverte, tous les timbres sur briques retrouvés à Rome et sur son territoire.

18 Le catalogue des timbres de Portus comprend pour l'instant environ 700 exemplaires dont environ un tiers doivent encore être identifiés : une trentaine 
provenant du môle nord-sud, une trentaine de la zone de l'Antemurale, une cinquantaine du môle de la Lanterne, une centaine de la basilique, près de 150 des entrepôts dits de Trajan et près de 380 de la zone du Palazzo Imperiale. Le travail d'identification des timbres sera complété en 2019 et la version définitive du catalogue devrait être prête pour la fin du quinquennal en 2021.

\section{NOTES}

1. Nous rappelons que cette étude s'insère dans le programme quinquennal de l'EFR (2017-2021) intitulé «Ostie-Portus, hub de l'empire romain » ainsi que dans le cadre du protocole d'accord signé le 12 avril 2019 entre le Parco Archeologico di Ostia Antica et l'EFR, la British School at Rome, le département d'archéologie de l'Université de Southampton et l'Université de Huelva. Les résultats de la première campagne sont disponibles en ligne à ce lien: https:// journals.openedition.org/cefr/2154

2. En dehors des grands môles en arc de cercle qui délimitaient le vaste bassin du port de Claude et qui ont été construits en eau à l'aide d'une armature en bois.

3. Voir le résumé de leur intervention intitulé «Considerazioni preliminari sulle fase edilizie del c.d. Molo della Lanterna e del paleoambiente circostante " lors du dernier séminaire ostiense (10-11 avril 2019) : https://www.ostiaantica.beniculturali.it/ups/2019/06/14/20190531-riassuntisesto-seminario-doc000614.pdf

4. L'USM 17 a été dégagé dans la limite de sécurité imposée par la dimension réduite du sondage C1 mais il n'est pas impossible que cette structure se poursuive plus en profondeur, en dessous de la dernière unité stratigraphique identifiée (US 50).

5. L'étude du matériel céramique provenant des différents sondages réalisés en 2018 est actuellement en cours d'étude par Marta Fedeli et un mémoire de laurea triennale est en cours de rédaction par Francesca Galanti, sous la direction d'Emanuela Borgia (Sapienza Università di Roma), sur le thème spécifique de « La ceramica fine e da mensa proveniente dagli scavi del molo nord-sud di Portus (EFR, Campagna 2018)».

6. L'US 31 en particulier se présente comme un dépôt sableux contenant une importante quantité de coquillages et d'algues décomposées.

7. Un mémoire de tesi triennale sur le thème des constructions portuaires a été entrepris par Jorge Cocquyt (Infrastrutture portuali nel Mediterraneo in epoca imperiale: alcune esemplificazioni), sous la direction d'Emanuela Borgia (Sapienza Università di Roma).

8. Dans une de ces cavités, a été déposée durant l'antiquité tardive une lampe à huile en terre cuite.

9. Après une interruption en 2019, les deux dernières campagnes de terrain du projet quinquennal (2020 et 2021) seront consacrées à l'étude de cette partie essentielle du môle, construit dans la continuité fonctionnelle et monumentale du portique de Claude, ainsi que des petits thermes tardifs qui s'y adossent sur sa façade portuaire.

10. Deutsches Archäologisches Institut. 
INDEX

Index géographique : Portus

Mots-clés : Portus, port, môle, quais, Rome, Claude

institutions École française de Rome, Parco Archeologico di Ostia Antica

\section{AUTEURS}

ÉVELYNE BUKOWIECKI

École française de Rome

\section{MILENA MIMMO}

Centre Camille Jullian

\section{CHARLES-ÉDOUARD SAUVIN}

Centre des Monuments Nationaux 